Ann. Biol. anim. Bioch. Biophys., I975, 15 (3), 495-50I.

\title{
COMPARAISON DES RELATIONS MÈRE-JEUNE OBSERVÉES LORS DE LA TÉTÉE CHEZ LA BREBIS (OVIS ARIES) ET CHEZ LA VACHE (BOS TAURUS)
}

\author{
P. POINDRON et P. LE NEINDRE* \\ Laboratoive du Comportement animal, \\ Station de Physiologie de la Reproduction, \\ Centre de Recherches de Tours, I. N.R. A., \\ Nouzilly, 37380 Monnaie, B.P. 1 \\ * Laboratoire de la Production de Viande, \\ Centre de Recherches de Clermont-Ferrand, I. N.R.A., \\ Theix, Saint Genès Champanelle, 63110 Beaumont
}

\section{RÉSUMÉ}

5 brebis et 4 vaches allaitant chacune un jeune ont été observées dans des stabulations libres. Lors de l'allaitement, 3 actes sont visibles, le passage au poitrail qui est un mouvement dirigé vers l'avant de la mère, le flairage ou le léchage (ce dernier uniquement chez la Vache), enfin la tétée avec trois positions possibles. La fréquence de ces actes ainsi que leur organisation en séquences sont très différentes chez les deux espèces ; le flairage de même que le passage au poitrail sont beaucoup plus fréquents chez la Brebis. Ceci pourrait traduire des différences dans les sens impliqués dans la reconnaissance des jeunes par la mère, l'olfaction étant le sens le plus important pour la Brebis, ce qui n'est pas le cas pour la Vache.

L'analyse comparative des comportements, déjà préconisée par l'entomologiste FABRE, a été depuis largement reprise. Plusieurs études ont été effectuées chez les insectes (RoTHENBUHLER, I964 ; VANCASSEL, I974). De même, des similitudes comportementales ont été observées dans plusieurs espèces de Vertébrés, notamment d'Oisealx (Heinroth, igil ; Whitman, igi9; Tinbergen, I959; Von De Wall, I963 ; etc.).

Une telle approche comparative est intéressante, car elle permet d'étudier comment deux espèces peuvent utiliser un patrimoine comportemental commun. Il est alors possible de mieux apprécier la valeur adaptive d'un comportement donné. 
Nous avons cherché dans le présent travail à comparer les manifestations de la relation mère-jeune lors de la tétée chez deux espèces d'Ongulés domestiques : la Brebis (Ovis aries) et la Vache (Bos taurus).

\section{I. - MATÉRIEL E'T MÉTHODES}

Les observations se sont déroulées séparément sur les Ovins au C. R. V. Z. de Nouzilly et les Bovins au C. R. Z. V. de Theix. C'est pourquoi les méthodes d'étude sont présentées à part.

\section{A. - Procédé d'étude des relations mère-jeune chez la Brebis}

Les brebis observées sont cinq femelles multipares de race Ile-de-France. Elles se trouvent en bergerie et disposent de $1,5 \mathrm{~m}^{2}$ par mère. Les ovulations ont été synchronisées par action d'un progestagène et les parturitions par injection de déxaméthasone (Bosc, 1972). Toutes les naissances ont ainsi été réparties dans une période d'environ 60 heures, ce qui assure une bonne homogénéité dans l'âge des agneaux.

Les brebis élèvent chacune un seul jeune et se trouvent dans un lot de ro femelles allaitantes. L'observateur se tient sur une plate-forme à l'extérieur du parc.

Chaque couple mère-jeune est observé une heure par jour et 5 jours par semaine, à partir du cinquième jour de la vie des agneaux, et pendant 3 semaines, soit 75 heures au total.

\section{B. - Procédé d'étude des relations mère-jeune chez la Vache}

4 vaches multipares de race Charolaise élevant un seul veau sont observées dans deux cases de $20 \mathrm{~m}^{2}$. Les vêlages se sont étalés sur une semaine.

L'observateur se tient à l'extérieur des cases. Chaque mère est observée 4 heures par jour pendant i i jours répartis uniformément au cours du premier mois de la vie du jeune, soit I 76 heures au total.

Nous avons considéré au cours de ce travail qu'il y a tétée lorsque le jeune prend un trayon dans la gueule. La fin de la tétée est marquée par le déplacement de la mère ou du jeune.

Lors d'un allaitement, la mère et le jcune effectuent des actes qui se succèdent à des intervalles de temps courts de quelques secondes. La séquence comportementale de tétée est l'enchaînement de tous les actes effectués par la mère et le jeune en rapport avec une tétée.

\section{II. - RÉSULTATS}

Avant d'analyser la séquence elle-même, nous décrirons rapidement ses actes, dont la plupart sont communs aux deux espèces étudiées.

\section{A. - Description des interactions}

Trois éléments comportementaux sont communs aux deux espèces :

\section{Passage au poitrail.}

Souvent le jeune, avant de se diriger vers la mamelle va vers la tête de sa mère, passe sous le cou ou s'arrête au niveau de l'épaule et, ensuite, essaie de téter. Cet acte est donc un mouvement, dirigé vers l'avant de la femelle. 
Positions d'allaitement.

Au cours de la tétée, le jeune accède à la mamelle dans l'une des trois position suivantes (fig. I) :

- le corps en position parallèle inversée à celui de la mère ( $\mathrm{PA}_{0}$ dans les figures) ; cette position est la plus fréquente chez les Ongulés (Frazer, I968);

- le corps du jeune en position perpendiculaire à celui de la mère $\left(\mathrm{PA}_{\mathbf{1}}\right)$;

- la tête du jenne entre les pattes arrière de la mère : position arrière $\left(\mathrm{PA}_{2}\right)$.
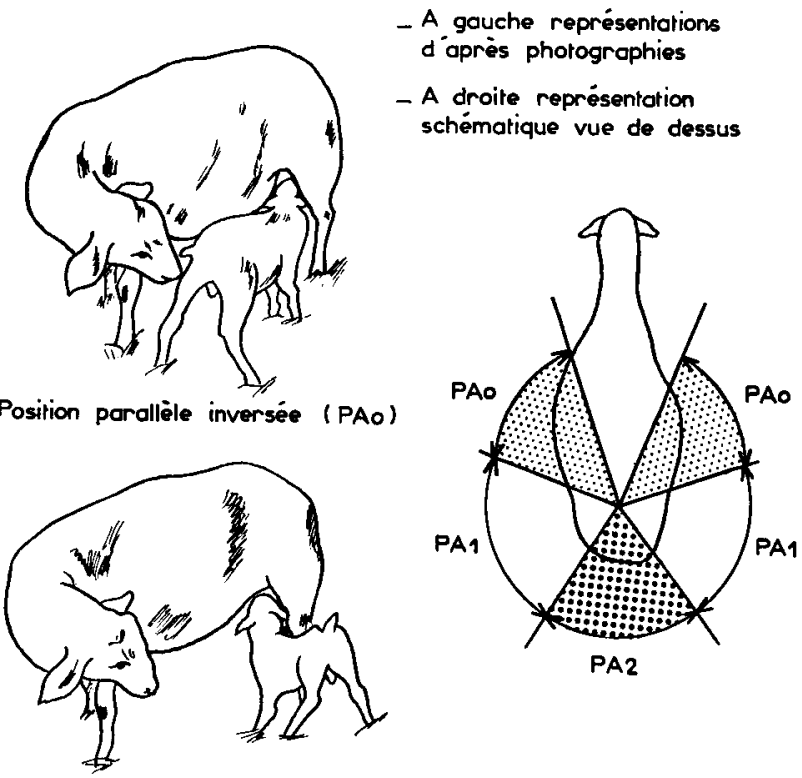

Position perpendiculaire (PA1)

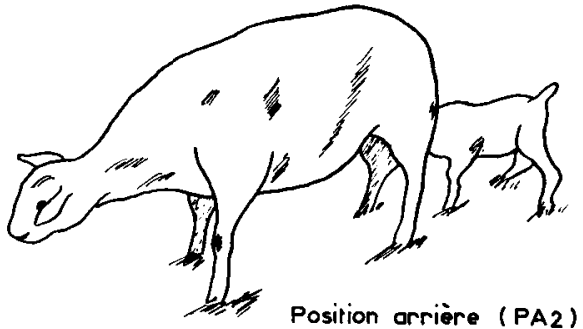

Fig. I. - Positions adoptées par la mère et le jeune lors de l'allaitement chez les Ovins. Les postures sont identiques dans l'espèce bovine

\section{Flairage.}

L'odorat joue un rôle important chez les Ongulés. Dans une étude comportementale, seuls les éléments moteurs peuvent être observés objectivement : nous avons considéré qu'il y avait flairage lorsque l'animal approche le nez à moins de $\mathrm{I} o \mathrm{~cm}$ du sujet flairé.

Au cours de nos observations lors de la tétée, seuls lesflairages du jeune par la mère ont été analysés. En effet, les flairages effectués par le jeune sont très rares à ce moment. 
Cependant, outre le passage au poitrail, la prise d'une position d'allaitement et le flairage, on observe en plus, deux types d'interactions chez la Vache :

- les léchages du jeune par sa mère sont fréquents chez les Bovins. Chez la Brebis, au contraire, ils sont limités aux quelques heures qui suivent la parturition;

- les émissions sonores en cours de tétée sont exceptionnelles chez les Ovins alors que, pendant l'allaitement, elles existent chez les Bovins (I4 p. Ioo des tétées).

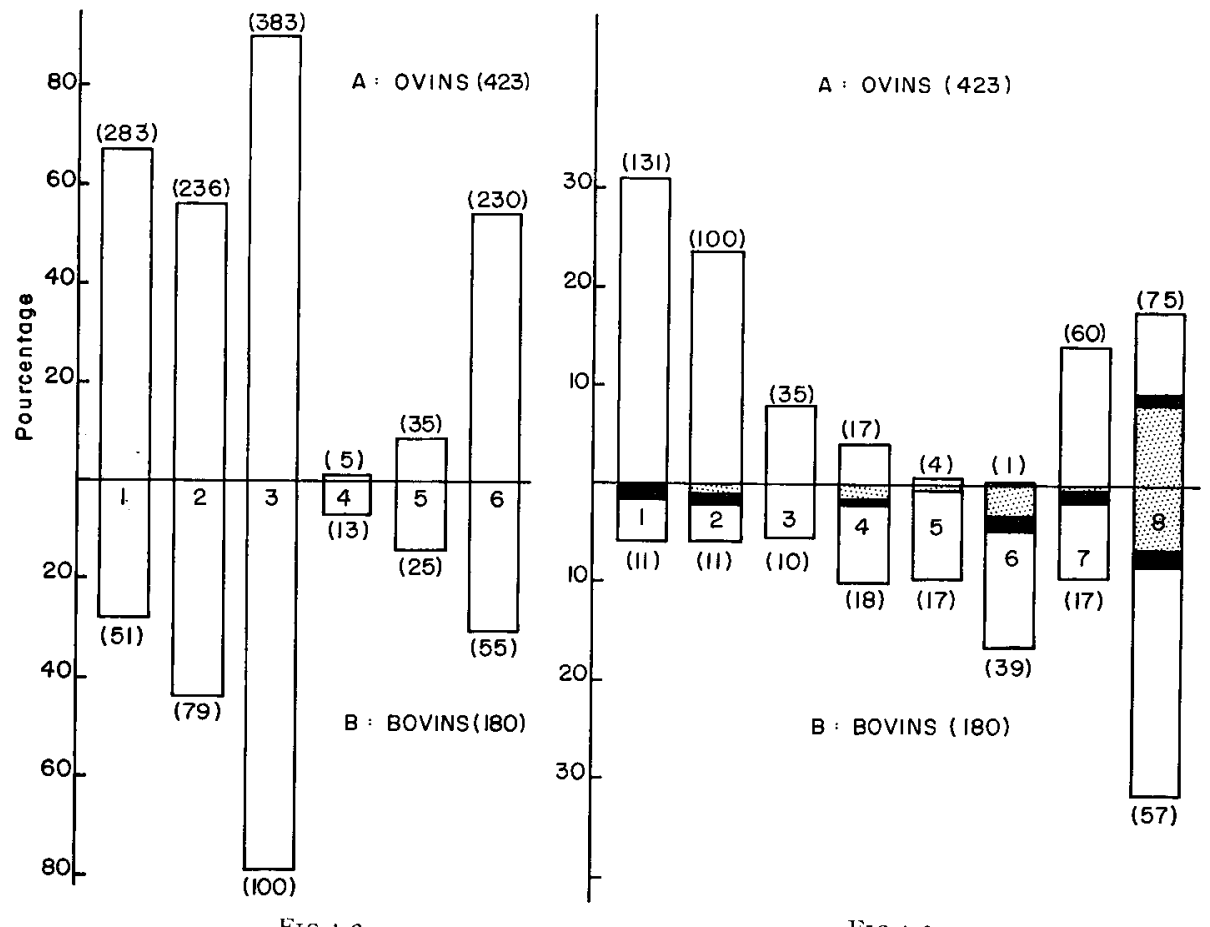

FIG : 2

FIG : 3

FIG. 2. - Fréquence de différents actes

pendant la séquence d'allaitement chez des Bovins et des Ovins

(Les nombres entre parenthèses indiquent le nombre d'observations)

I : Passage au poitrail

Flairage du jeune avant le début de l'allaitement

Position d'allaitement parallèle inversée

Position d'allaitement perpendiculaire

Position d'allaitement arrière

6 : Flairage du jeune après le début de l'allaitement.

FIG. 3. - Proportions des différentes séquences d'allaitement observées chez des Bovins et des Ovins

(Les nombres entre parenthèses indiquent le nombre d'observations)

$\mathrm{P} \quad$ : Passage au poitrail

$\mathrm{F}$ : Flairage (ou léchage pour les bovins)

$\mathrm{PA}_{0}$ : Position d'allaitement inversée (blanc)

$\mathrm{PA}_{1}$ : Position d'allaitement perpendiculaire (noir)

$\mathrm{PA}_{2}$ : Position d'allaitement arrière (gris)

De I à 8 : differents types de séquences observées
I P F PA F
3 P PA F
5 F PA F
7 PA F
2 P F PA
4 P PA
6 F PA
8 PA 


\section{B. - Analyse des séquences de tétée observées chez la Vache et la Brebis}

Lors de la tétée, les différents actes s'organisent en séquences dont l'analyse permet de mieux comprendre quelles sont les informations échangées par les animaux à ce moment.

I. Chez la Brebis, 67 p. Ioo des séquences de tétée comprennent un passage au poitrail de la part du jeune ; chez la Vache, ce comportement n'est observé que dans 28 p. Ioo des cas ( $p<$ o,or ; fig. 2$)$.

2. $7^{8} \mathrm{p}$. Ioo des tétées comprennent un flairage de l'agneau par la mère ; cette proportion n'est que de 59 p. Ioo chez la Vache ( $p<0$,or ; fig. 2).

Chez la Brebis, le flairage du jeune avant la prise d'une position d'allaitement est pratiquement toujours associé à un passage au poitrail ( $98 \mathrm{p}$. Ioo des cas) ; par contre, chez la Vache, lorsque le jeune est léché ou flairé avant la prise d'une position d'allaitement, il n'y a que dans 29 p. Ioo des cas passage au poitrail. I a liaison entre les deux comportements apparaît donc plus faible chez les Bovins que chez les Ovins $(p<0$, or $)$.

3. Ia position d'allaitement parallèle inversée est plus fréquente chez la Brebis (90 p. Ioo des cas) que chez la Vache (79 p. Ioo des cas ; $p<0$,or).

L'étude des séquences de tétée, présentée dans la figure 3 , montre l'existence, chez la Brebis, d'une séquence plus fréquente que les autres et qui comprend le passage au poitrail $(\mathrm{P})$ accompagné d'un flairage $(\mathrm{F})$ par la Brebis, ainsi que la prise d'une position d'allaitement parallèle inversée $\left(\mathrm{PA}_{0}\right)$ (séquences $\mathrm{n}^{\circ} \mathrm{I}$ et $\mathrm{n}^{\circ} 2$ de la figure $3 \mathrm{~A}$ ). Par contre, chez la Vache, la séquence la plus fréquente est la tétée en position parallèle inversée $\left(\mathrm{PA}_{0}\right)$, sans passage au poitrail, ni flairage (séquence no 8 de la figure $3 \mathrm{~B}$ ). Assez souvent, il y a un flairage sans passage au poitrail suivi d'une tétée en position parallèle inversée (séquence 6 de la figure $3 \mathrm{~B}$ ). Les autres séquences sont pratiquement équiprobables.

Les séquences P-F-PA $A_{0}$, fréquentes chez la Brebis, ne sont donc qu'accessoires chez la Vache.

\section{III. - DISCUSSION E'T CONCLUSION}

Il apparaît donc que le jeune Veau et le jeune Agneau montrent des comportements identiques. La prise d'une position de tétée parallèle inversée existe dans les deux espèces ainsi que chez la plupart des Ongulés, exceptés ceux qui allaitent couchés tels que la Truie.

De même, le passage au poitrail s'observe aussi bien chez le Veau que chez l'Agneau. Il est connu également chez le Poulain (W. Martin-Rosset, communication personnelle), chez le Chevreau (observations personnelles) et chez le Mouflon (ovis musimon), pour lequel GEIST (I97I) décrit un "run-around » de la part du jeune : celui-ci court autour de sa mère et passe devant elle avant de téter.

L'existence de comportements communs observables très rapidement après la naissance, dans plusieurs espèces d'Ongulés, correspond à un patrimoine héréditaire communn à ces espèces. 
Cependant, les manifestations comportementales ne sont pas pour autant identiques dans les espèces envisagées : chez la Brebis, il existe une séquence de tétée "préférentielle " qui est absente chez la Vache. I1 a été montré chez la Brebis (PonNDRON, I974) que cette séquence dépend étroitement du comportement maternel sélectif de la Brebis, lequel repose sur des critères essentiellement olfactifs (Bouissou, I968; MoRGAN, I970). I,e passage au poitrail favorise la reconnaissance de l'agneau par sa mère en facilitant son flairage. Cette reconnaissance préalable facilite l'acceptation du jeune à la tétée (PoINDron, I974). L'Agneau met en place le comportement le mieux adapté à la réussite de la tentative de tétée : la suppression de la sélectivité de la mère supprime l'apparition d'une séquence de tétée préférentielle. Chez la Vache, on n'assiste pas à la mise en place de façon aussi fréquente du passage au poitrail : celui-ci, bien que présent dans le " répertoire " comportemental du Veau, ne semble pas posséder le même caractère adaptatif que chez l'Agneau. Deux faits appuient tune telle hypothèse :

- D’une part, la reconnaissance préalable du jeune Veau avant la tétée ne nécessite pas de façon absolue 1'existence du passage au poitrail puisque 7 I p. Ioo des léchages ou flairages apparus avant la prise d'une position de tétée sont apparus sans le passage au poitrail (alors que chez la Brebis, ce type de flairage n'est pas associé au passage au poitrail que dans $4 \mathrm{p}$. roo des cas).

- D'autre part, si la reconnaissance du jeune est essentiellement olfactive chez la Brebis, il n'en est probablement pas de même chez la Vache. Des travaux récents (LE NEINDRE, non publiés) ont montré que des vaches privées de la vue par masquage refusent, de la même façon que lorsqu'elles sont normales, de se laisser téter par un veau étranger. Ces mêmes vaches, non masquées, refusent les veaux étrangers enduits d'huile de pied de bœuf, produit qui semble perturber la reconnaissance olfactive. Par contre, lorsque les mères sont masquées et que simultanément les jeunes étrangers sont enduits d'huile de pied de bœuf, dans 50 p. Ioo des essais, les vaches se laissent téter.

Chacun de ces deux sens, vue et odorat, suffit donc pour discriminer les veaux. Il faut des masquages visuel et olfactif simultanés pour empêcher la reconnaissance du Veau par la Vache.

Reçu pour publication en mars 1975.

\section{REMERCIEMENTS}

Ce travail a été réalisé dans le cadre de 1'A. T. P. - - I. N. R. I 1 10 $055^{2} 8$.

\section{SUMMARY}

COMPARATIVE STUDY IN THE EWE (OVIS ARIES)

AND THE COW (BOS TAURUS) OF THE BEHAVIOURAL INTERACTIONS BETWEEN THE MOTHER AND THE YOUNG AT SUCKLING

The behavioural interactions between the mother and the young were observed in 5 ewes and 4 cows. The three following patterns occur in both species : a movement of the young in the direction of the anterior part of the dam's body -- sniffing at the young (and licking in the case of the 
cow) - suckling that can take place in one of three positions : reverse parallel (normal), perpendicular or from behind. The absolute frequency of such patterns and their organisation in a sequence are different between the two species : the movement to the anterior part of the dam's body and the sniffing of the young are more frequent in the ewe as compared to the cow. This may result from differences in the sensory informations allowing the individual recognition : in the sheep, sniffing is critical for the acceptance or refusal of the lamb attempting to suckle, and the movement of the young facilitates the control by the dam. In the cow, however, the sense of smell does not appear to have such a critical importance in the identification of the calf.

\section{RÉFÉRENCES BIBLIOGRAPHIQUES}

Bosc M. J., I972. The induction and synchronisation of lambing with the aid of dexamethasone. $J$. Reprod. Fert., 28, 347-357.

Bouissou M. F., I968. Effet de l'ablation des bulbes olfactifs sur la reconnaissance du jeune par sa mère chez les ovins. Rev. Comp. Anim., 3, 77-83.

FRAZER A. F., I969. In reproductive behaviour in Ungulates, Academic Press, London, I32-I53.

Heinroth O., I9II. Beitrage zur Biologie Namentlich Ethologie und Psychologie der Anatiden. Verhandlungen Intern. Ornith. Kongress., 589-702.

Morgan P. D., I970. Maternal behaviour in the ewe Ph. D. Univ. Western Australia.

PoIndron P., I974. Contribution à l'étude éthologique de la relation mère-jeune chez les ovins domestiques (Ovis aries L.). Thèse, Université de Provence.

Rothenbuhler W. C., I964. Behaviour genetics of nest cleaning in honey-bees I. - Responses of four inbred lines to disease killed brood. Anim. Behav., 12, 578-583.

Tinbergen N., r959. Comparative studies of the behaviour of Gulls (Laridae) : a progress report. Behaviour, 15, 1-70.

VAncassel M., Caussanel C., rg68. Contribution à l'étude descriptive du comportement de Labidura riparia L. (Labiduridae, Dermaptère). Rev. Comp. Animal, 2, I7 p.

VON DE WALl W., r968. Le comportement des canards hybrides. Ann. Soc. R. Zool. Belg., $98,125$.

Whitman C. O., rgig. The behaviour of Pigeons. Carnagie Inst. Wash., Publ., 257, I-I6I. 